book as anatomically-orientated because it differs from organism-orientated works such as Odds' Candida and candidosis, or the disease-oriented Candidiasis by Bodey and Fainstein, both of which are broader in their coverage of the organism (Odds) or its disease involvement (both books). It is difficult to describe candidosis as anything other than a phenomenon. As the book emphasises, this is not simply an infection, a disease, or a syndrome, but a continuum from harmless commensalism to an invasive and life-threatening infection. A diagram indicates that oral candidosis results only when a limited combination of host factors, organism factors and environmental factors are present. The latter are perhaps the most loosely described of the three, and some might question just how important environmental factors are in comparison with the other two.

The mouth is an important and well-described site of Candida residence and infection, and the book serves a useful purpose in reviewing the oral aspects of the organism. Thus, it is particularly valuable to dental practitioners, oral pathologists and oral microbiologists. It contains several chapters reviewing the biology and disease-related aspects of the organism which are of general interest to microbiologists. There is also a useful review of oral immunology and its role in oral candidosis. The other microbiologically important chapters are concerned with laboratory diagnosis and with antifungal agents. Again these are useful general reviews for the non-expert in these fields. Personally, in the chapter on laboratory diagnosis, I would have preferred to see slightly more emphasis on quantitative aspects of isolation and perhaps less concern with sensitivity of detection of the organism, acknowledged to be present in small numbers in most of us.

The largest section of the book is concerned with the diseases, pathological changes and clinical aspects of oral candidosis. While of interest to the general microbiologist, these matters are covered essentially for a different readership from those of this Journal. The material is logically arranged, well-illustrated with some excellent colour photographs, and clearly forms a very useful review for the dental practitioner or oral pathologist.

Overall the book is is a useful addition to the shelves of all who have a deep interest in candida disease, whether specifically oral or otherwise. However, it is a somewhat expensive book and, with its emphasis primarily on the clinical aspects of disease, it will perhaps be a library reference book rather than a personal purchase for most microbiologists.

\section{W. PenN}

\section{Lyme Borreliosis II}

Edited by G. StaneK, W. Kristoferitsch, M. Pletschette, A. G. BARbOUR and H. Flamm. 1989. Gustav Fischer Verlag, Stuttgart, New York. Pp 365. DM 180.

The increased awareness of the significance of Lyme borreliosis worldwide has prompted the organisation of a series of international symposia on the epidemiology, diagnosis and pathogenesis of Borrelia burgdorferi infections. This volume is based on selected papers presented at a conference entitled "Lyme Borreliosis Update Europe" held in Vienna in 1987, and also contains reports on two WHO workshops of Lyme borreliosis in Europe held at the above meeting and a subsequent meeting in Prague in 1989.

The data are presented in the form of short papers and cover diverse areas of interest including classification of $B$. burgdorferi, insect vectors and transmission, epidemiology, pathogenesis, clinical features, therapy and serodiagnosis. Of necessity, the work included in each chapter is selective and for the most part does not attempt to review particular subject areas as a whole. In addition, several chapters draw together disparate areas of study and inclusion of a brief introduction to highlight the common theme would have been beneficial in some cases. This has been done in the chapter on clinical features of the disease and helps the presentation considerably. This chapter and those on therapy and serodiagnosis are particularly well presented and contain information which may be valuable for clinicians and laboratories involved in the diagnosis of $B$. burgdorferi infections. The incidence of Lyme borreliosis in various European countries and, presumably for comparison, China and Africa is discussed in the epidemiology chapter which, surprisingly, contains no information on the incidence or distribution of these infections in the UK. This may reflect the availability of information on this subject in 1987 and highlights a general criticism of the text in that some of the data presented may now be out of date or may have been superseded by subsequent publications. The latter may also explain the apparent bias of the section on pathogenesis which concentrates almost exclusively on host factors in relation to infection and only briefly considers virulence determinants of the bacterium in the infectious process, an area which has received more attention in the recent literature.

The reports of the WHO workshops provide useful information on the global impact of Lyme borreliosis and highlight areas of the disease and its diagnosis that merit further attention. A list of contact addresses of individuals working on these infections in Europe is also included.

Overall, this text should provide information of general interest to those working on Lyme borreliosis throughout Europe, particularly in relation to the clinical and serological diagnosis of this increasingly important disease. However, it is not sufficiently up to date or comprehensive to be recommended as a definitive text.

A. COCKAYNE

\section{A Textbook of Parasitology}

SubHash S. KelKar \& Rohini S. KelKar. 1990. Sangam Books. Pp. 176. £6.95.

This textbook of parasitology is a slim paperback volume. It is intended for undergraduate medical students especially for the Indian environment. It contains line drawings and some photographs but I am afraid that these have not reproduced well. Furthermore, the book is printed on rather thin paper which looks as though it will age rapidly.

The book covers all the topics that might be expected in such a text. The authors clearly have extensive experience of parasitology in India, and some sections are very well done. Other newer developments are less well covered. For example, Cryptosporidium, a major cause of gastroenteritis world-wide, merits less than a page, whereas Enterobius gets a page and a half. The sections are all organism based rather than on organ systems or disease syndromes, but perhaps parasitic disease is less easily dealt with according to clinical syndromes. There is not too much attention to taxonomy and in some cases immunity to infection is also covered. I would have liked to see a little more coverage of pathogenesis and pathogenic mechanisms.

I am not sure that this book would be recommended for medical students in the UK.

\section{A. HART}

\section{CRC Handbook of laboratory safety}

Edited by A. KeITH FurR. 1990. CRC Press, Inc. Boca Raton, Florida. Pp. 704. $£ 76.00$.

The stated aim of this book is to guide research personnel in the implementation of effective health and safety programmes in their facilities. The 19 contributors are 
American, and it is written primarily for the USA market where the potential for litigation has resulted in meticulous attention to safety law, and the need for equally detailed guidance towards its interpretation. The previous edition of this Handbook was published in 1971 and an update is now welcome. The advent of AIDS and other newly recognised diseases, together with improved knowledge of the carcinogenic and teratogenic effects of certain substances, has necessitated the inclusion of much new material.

The book covers the assignment of responsibilities and organisation of laboratory safety in a small introductory section and offers excellent advice. There are long and detailed chapters on laboratory design, equipment, and operations, together with more specific information for routine and individual areas including radio-isotope, laser, $\mathrm{X}$-ray, biomedical and recombinant DNA laboratories.

At 704 pages, mostly of closely written text, this is probably not a volume to read from cover to cover, unless one is setting up a Safety Committee with a wide ranging remit, or taking on the responsibility for the safety aspects of a new laboratory. There are, however, particular sections that will serve to enhance the appreciation of safety problems for any laboratory worker. Unfortunately while this may be the definitive work for the USA, our laws are not identical, and UK readers may still need to refer to other sources even if they have access to this volume. For two countries divided by a common language, the text of this work is clear to understand, but the liberal use of unfamiliar acronyms, OSHA, SARA, ERP, NIOSH and many others is irritating until they are learnt. No doubt the same could be said of COSHH and RIDDOR if they were to appear in the USA.

As a quick reference work this book is inappropriate, but for those seeking more detailed information and ideas regarding the solution to safety problems in the laboratory it is recommended as a source of practical knowledge. It would be a valuable addition to the library of a group of laboratories but is too broad in its content to justify the cost of $£ 76.00$ for individual departments with limited budgets.

D. E. Healing

\section{Clinical and Molecular Aspects of Anaerobes}

Edited by S. P. BorRIELlo. 1990. Blackwell Scientific Publications, Oxford. Pp. 329. £39.50.

This book comprises the proceedings of the Sixth International Symposium of the Anaerobe Discussion Group, a group of microbiologists with an interest in anaerobic microbiology.

The book, as was the Symposium, is divided into seven different aspects of anaerobic microbiology: anaerobic mycoplasmas and protozoa; anaerobic infections in paediatrics; anaerobes and food poisoning; nutrient availability and effects on anaerobes; virulence factors of anaerobes; molecular studies on anaerobes; and laboratory diagnosis and taxonomic considerations. The topics covered in each section include "invited overview presentations", free papers, abstracts of posters, even case reports. The range of subjects obviously reflects, to a large extent, the interests of those attending the meeting; it does not cover all aspects of anaerobic microbiology.

The overview presentations, especially those on infant botulism, nucleic acid probes, virulence factors in anaerobes and colonisation resistance, are excellent and form a useful update on knowledge in these areas although some of the other contributions are rather brief and lack any technical details. The section on anaerobic mycoplasmas and protozoa was also of a high quality and provided a fascinating insight into an aspect of anaerobic microbiology that is seldom considered by "anaerobiologists", and seldom discussed in textbooks.

One of the most interesting contributions is that on "the rapid diagnosis of anaerobic infection" by Phillips and Eykyn. This is full of useful information and common sense advice. The comment that "the collection of a good specimen alone, involving the release of pus, provided diagnosis and complete therapy in many cases" is one that should be emphasised strongly to all microbiologists-and surgeons. All too often, anaerobic infections that require incision and drainage are treated by antibiotics alone. One minor pointreference 17 is missing in this chapter.

The changing taxonomy of anaerobes is difficult to keep abreast of. Articles that attempt to keep the reader up to date are welcome but the paper on "current taxonomic status of the genus Bacteroides" was disappointingly short-the authors should have been asked to write a full review. I am prejudiced-but, with the exception of the very good paper by Murdoch, there was little mention of anaerobic cocci in this book.

This is a well-edited book, well produced and with few errors. It is a fascinating source of information. By its nature, it has only limited appeal to a general readership, but I have no doubt that it will be required reading for all those with an interest in anaerobic microbiology.

B. WATT 